\title{
EVALUACIÓN DE LAS CONCEPCIONES DE ESCRITURA ACADÉMICA EN DOCTORANDOS EN EDUCACIÓN
}

EVALUATING DOCTORAL STUDENTS' CONCEPTIONS ON ACADEMIC WRITING

\section{Volumen 13, Número 3}

Setiembre - Diciembre

pp. 1-21

Este número se publicó el 30 de setiembre de 2013

Hilda Difabio de Anglat

Revista indizada en REDALYC, $\underline{\text { SCIELO }}$

Revista distribuida en las bases de datos:

CATÁLOGO DE LATINDEX, IRESIE, CLASE, DIALNET, DOAJ, E-REVIST@S, SHERPA/ROMEO, QUALIS, MIAR

Revista registrada en los directorios:

ULRICH'S, REDIE, RINACE, OEI, MAESTROTECA, PREAL, CLASCO 


\title{
EVALUACIÓN DE LAS CONCEPCIONES DE ESCRITURA ACADÉMICA EN DOCTORANDOS EN EDUCACIÓN EVALUATING DOCTORAL STUDENTS' CONCEPTIONS ON ACADEMIC WRITING
}

\author{
Hilda Difabio de Anglat ${ }^{1}$
}

\begin{abstract}
Resumen: El propósito de este artículo es presentar una escala orientada a evaluar las concepciones de escritura académica de estudiantes de doctorado y a ponderar las cualidades psicométricas -confiabilidad y discriminación de los ítems- que habilita el tamaño de la muestra. Se conforma de 33 ítems referidos a la naturaleza y funciones de la escritura y a las estrategias recursivas de planificación, textualización y revisión: 24 traducidos y adaptados de instrumentos estandarizados y 9 desarrollados ad hoc. Estos últimos se distribuyen en dos subescalas: escritura como Elaboración (17 ítems) y como Reproducción (16 ítems). En una muestra piloto de 39 doctorandos en Educación de Argentina (Universidad Nacional de Cuyo, Mendoza, y Universidad Católica de Cuyo, sedes San Juan y San Luis) se aplica el instrumento en forma auto-administrada, vía correo electrónico, previo consentimiento del alumnado. La escala muestra índices considerables de confiabilidad según el alfa de Cronbach: un coeficiente de 0,93 para la subescala Elaboración y de 0,91 para la escritura como Reproducción. También, evidencia que resultan discriminativos todos los ítems de la primera y el 87,5\% de los ítems de la segunda. Su potencial utilidad reside en que las concepciones de escritura, un área de investigación de escaso desarrollo, ejercen un fuerte papel mediador en los procesos cognitivo-lingüísticos, en las actitudes hacia la escritura y en la motivación, los cuales -a su turno- influyen en las actividades escriturales. De allí la necesidad de que el doctorando sustituya el enfoque superficial ( $41 \%$ en nuestra muestra) por el profundo.
\end{abstract}

Palabras clave: ESCRITURA ACADÉMICA, CONCEPCIONES DE ESCRITURA, ESTUDIANTE DE DOCTORADO, CIENCIAS DE LA EDUCACIÓN, CONSTRUCCIÓN DE ESCALAS DE MEDICIÓN, ARGENTINA

\begin{abstract}
The purpose of this paper is to present a scale aimed to assessing the conceptions on academic writing of PhD students and to weight the psychometric qualities -reliability and discrimination of items- that enables sample size. It is made up of 33 items related to the nature and functions of writing and to the recursive strategies of planning, textualization, and revision: 24 translated into Spanish and adapted from standardized instruments, and 9 developed ad hoc. This set is divided into two subscales: writing as Elaboration (17 items) and as Reproduction (16 items). Using a pilot sample of 39 PhD students in Education of Argentina (Universidad Nacional de Cuyo, Mendoza, and Universidad Católica de Cuyo, at San Juan and San Luis), the instrument was selfadministered, via email, with prior consent of the students. It showed considerable reliability indices according to Cronbach alpha: a coefficient of 0.93 for Elaboration subscale and of 0.91 for writing as Reproduction. It also showed that all the items of the first and $87.5 \%$ of the second are discriminative. Its potential usefulness is that conceptions on writing, a research area of poor development, have a strong mediating role in cognitive-linguistic processes, attitudes towards writing, and motivation; which also affect writing activities. Hence the need for doctoral students to replace the surface approaches $(41 \%$ in our sample) by the deep one.
\end{abstract}

Key words: ACADEMIC WRITING, CONCEPTIONS ON WRITING, DOCTORAL STUDENT, EDUCATIONAL SCIENCES, MEASUREMENT SCALES DEVELOPMENT, ARGENTINA

\footnotetext{
${ }^{1}$ Investigadora de CONICET (Consejo Nacional de Investigaciones Científicas y Técnicas), con sede en el Centro de Investigaciones Cuyo, Mendoza, Argentina. Doctora en Ciencias de la educación.
}

Dirección electrónica: centroinvestigacionescuyo@speedy.com.ar; ganglat@gmail.com

Artículo recibido: 18 de marzo, 2013

Aprobado: 12 de setiembre, 2013 


\section{Introducción}

La alfabetización académica (academic literacy en lengua inglesa) o literacidad académica (Cassany y Morales, 2009) -los modos de buscar, elaborar y comunicar conocimiento mediante la escritura-, es la actividad prioritaria de un doctorado; dado que su producto final es una Tesis, esto es, una exposición escrita que presenta el alumno ante la universidad, para la conclusión de sus estudios, que permite demostrar que amerita el grado académico al que aspira, porque, a través de este trabajo, se presenta como un estudioso capaz de hacer avanzar la disciplina a la que se dedica. Su producción le exige al doctorando un salto cualitativo, una transición que se ha caracterizado como crítica: el paso desde "cursante de seminarios", esto es, un consumidor del conocimiento cuidadosamente distribuido en cursos, módulos y listas de lectura, a investigador (Lovitts, 2005), a productor de un conocimiento original, a "enunciador autorizado de cara a la comunidad científica de futuros pares" (Carlino, 2005, p. 52).

En este marco, la importancia de abordar las concepciones de escritura, un área de investigación de escaso desarrollo, reside en el fuerte papel mediador que estas ejercen en los procesos cognitivo-lingüísticos, en las actitudes hacia la escritura y en la motivación, los cuales -a su turno- inciden en las actividades escriturales. Si lográsemos comprender estas concepciones implícitas y distinguir los factores que las influyen, hallaríamos una ventana para mejorar las habilidades, las estrategias y las actitudes de los escritores de cualquier edad. En el nivel de posgrado, adquieren una relevancia especial porque la escritura demanda "una extensión, un nivel de conceptualización original, un grado superior de integración de conocimientos y una capacidad de autoorganización y regulación del trabajo autónomo que solo pueden vehiculizarse por medio de un dominio escritor difícilmente obtenido en etapas anteriores" (Narvaja de Arnoux et al., 2005, p. 3).

De allí que el presente trabajo persiga el propósito de presentar una escala de evaluación de las concepciones de escritura académica en el doctorado y ponderar las cualidades psicométricas -confiabilidad y discriminación de los ítems- que habilita el tamaño de la muestra.

\section{Referente teórico}

Inspirados en los estudios sobre las concepciones de los alumnos acerca del aprendizaje, los investigadores comenzaron a aplicar las mismas ideas a la escritura en el 
nivel universitario (Biggs, 1988; Hounsell, 1984; Lavelle, 1993) y en el posgrado (Biggs, Lai, Tang y Lavelle, 1999).

A partir del trabajo seminal de Marton y Säljö (1976), el término "concepciones" se adscribe a la descripción de la variación en las interpretaciones de los alumnos de sus experiencias de aprendizaje. Respecto de la escritura, se entienden como redes de supuestos, conocimientos y valoraciones acerca del proceso. Pero, las investigaciones son escasas y, como proceden de diversos marcos teóricos, "Ios supuestos sobre la naturaleza de las concepciones e incluso los propios términos para referirse a ellas son diferentes" (Villalón Molina, 2010, p. 77).

La distinción clave, propuesta por Biggs (1988) desde la fenomenografía, opone un enfoque profundo, significativo, de la escritura -basado en la percepción de la tarea como un compromiso complejo y proactivo- a un enfoque superficial, en el cual la intención es reproducir o reorganizar información sólo para alcanzar los requisitos. Posteriormente, se diferencian también en términos de foco: el primero implica una focalización alta de la atención en los procesos escriturales ${ }^{2}$, mientras que el segundo se queda en aspectos micro, tales como el léxico y la gramática (Lavelle y Guarino, 2003).

En los siguientes apartados, desde los tres enfoques -fenomenográfico, metacognitivo y del aprendizaje social- que distinguen Villalón Molina y Mateos (2009), abordaremos la categorización de las concepciones sobre la escritura y algunos resultados de la investigación empírica.

\subsection{Enfoque fenomenográfico}

La mayor parte de los estudios se han realizado desde este enfoque. El trabajo pionero de Hounsell (1984) sobre el ensayo halla tres concepciones cualitativamente diferentes en estudiantes de Historia: como argumento, como punto de vista y como disposición, arreglo. La primera, la más sofisticada, lo conceptúa como una presentación ordenada y coherente, que gira alrededor de una postura distintiva bien sustentada por la evidencia. El ensayo como punto de vista, si bien comparte el rasgo anterior de la alianza entre interpretación (significado o significados que el escritor otorga al material) y organización (como estructura integrada), no considera explícitamente el rol de la evidencia -aparecen referencias a los

\footnotetext{
2 Una secuencia de tres procesos recursivos según el modelo interactivo de Flower y Hayes (1981): planificación (pre-escritura: bosquejo, esquema, organizador gráfico, un índice comentado), puesta en texto, traslación o textualización y revisión desde una perspectiva psicolingüística, esto es, como una instancia de elaboración del significado, inherente al proceso de escritura.
} 
datos magras o indirectas-. En la última, se lo define sólo como una presentación ordenada de información discreta: se considera importante expresar todas las ideas, opiniones y datos -sobre todo cuantitativos-, pero no se advierte una preocupación concomitante por relacionar dichas ideas a fin de constituir un punto de vista unificado.

Campbell, Smith y Brooker (1998) implementan entrevistas con alumnos universitarios sobre su conceptualización del ensayo y las estrategias que emplean para elaborarlo; luego, evalúan el texto producido mediante tres categorías (uni-estructural, multi-estructural y relacional) de la Taxonomía SOLO (Structure of the Observed Learning Outcome) de Biggs y Collis (1982). Encuentran una asociación positiva entre concepciones constructivistas y ensayos más elaborados, por una parte, y entre concepciones más reproductivas y ensayos simplistas (los más frecuentes), por otra.

Si bien no se restringe al tema de las concepciones, el modelo de enfoques de escritura de Ellen Lavelle (1993) también se inscribe en esta línea fenomenográfica. Postula que las intenciones de los estudiantes cuando escriben condicionan su elección de las estrategias, las que a su vez afectan los resultados de la producción escrita. Desarrolla el Inventario de los Procesos de Escritura en la Universidad, Inventory of Processes in College Composition (Lavelle, 1993; Lavelle y Guarino, 2001; Lavelle y Zuercher, 2001), que el análisis factorial distribuye en cinco factores, dos de los cuales se relacionan con una concepción constructivista: Elaboración, la conceptualización de la escritura como una empresa personal profunda y el compromiso con dicha conceptualización, y ReflexiónRevisión, que también refiere a un proceso escritural profundo sustentado en la revisión como reformulación o reelaboración del propio pensamiento ${ }^{3}$.

En el posgrado, Lavelle y Bushrow (2007) distinguen siete enfoques, tres de los cuales son representativos de la escritura profunda: Elaborativo, Intuitivo -un escritor que muestra un ritmo y una fluidez más allá del plano cognitivo, en una conexión sensible que puede deberse a la lectura frecuente de artículos científicos que lo han familiarizado con el tempo de la escritura académica- y Científico, que cumple su rol pero necesita combinarse con

\footnotetext{
${ }^{3}$ Los tres factores representativos de la escritura superficial son: Baja autoeficacia, un enfoque altamente tímido basado en dudas sobre la habilidad y en la consideración de la escritura como una tarea penosa; Escala Espontánea-Impulsiva, un enfoque no planificado y a la vez defensivo por sobre-estimación de la habilidad y miedo de ocuparse en lo que el escritor percibe como limitaciones y Escala Procedural, estrategia derivada de una estricta adherencia a las reglas y con un mínimo compromiso.
}

Volumen 13, Número 3, Año 2013, ISSN 1409-4703 
otras estrategias a fin de no limitar la creatividad y la flexibilidad en tanto la escritura académica claramente excede a una estructura "bien afinada".

Por su parte, Ellis, Taylor y Drury (2007), a partir de un cuestionario conformado por dos escalas (cohesiva vs. fragmentada), investigan las concepciones de estudiantes universitarios de primer año sobre el aprendizaje de la Biología a través de la escritura. La escala cohesiva refiere a una relación estrecha con el contenido sobre el que se escribe, orientada a promover una comprensión profunda; la escala fragmentada, en cambio, asocia la experiencia escritural con aspectos superficiales y formales, como presentación y gramática. Los resultados muestran que los alumnos con una concepción cohesiva adoptan un enfoque profundo de aprendizaje, evidencian una estrategia sofisticada de revisión del texto y producen mejores informes científicos.

Boscolo, Arfé y Quarisa (2007) implementan una intervención pedagógica, también en alumnos de nivel universitario, orientada a mejorar la tarea de escritura de síntesis a partir de fuentes y a ponderar si esta incide en las creencias ${ }^{5}$ de los estudiantes sobre la escritura académica, evaluadas mediante una escala Likert en tres dimensiones: Elaboración y revisión del texto, Punto de vista personal -contribución idiosincrática del escritor en la tareay Transmisión del conocimiento. Hallan un incremento considerable de la competencia para producir una síntesis, pero ningún efecto del programa en la función elaborativa de la escritura, ya que sólo parece afectar la tercera dimensión de las creencias hacia su conceptualización como "impersonal".

\subsection{Enfoque metacognitivo}

Scardamalia y Bereiter (1992), a partir de la relación que establece el escritor entre el "espacio del contenido" (qué expresar) y el "espacio retórico" (cómo hacerlo), presentan su

\footnotetext{
${ }^{4}$ Los cuatro asociados a la escritura superficial son: No revisión; Baja autoeficacia; Orientado a la tarea, basado en supuestos lineales sobre la escritura a fin de terminarla -es el indicador más fuerte de un enfoque superficial de escritura en el nivel de posgrado- y Escultor, una estrategia descuidada, de "gran cuadro".

${ }^{5}$ Creencias y teorías implícitas son constructos alternativos a concepciones que algunos autores tratan como cuasi sinónimos y otros buscan distinguir desde sus diferentes tradiciones teóricas (temática que sin embargo excede los límites de este artículo). Las primeras se conceptualizan como construcciones psicológicas formadas por ideas, comprensiones, imágenes o proposiciones que se consideran verdaderas, dirigen las acciones y actúan como guías para la interpretación de situaciones nuevas, son representaciones más o menos implícitas que presentan una alta variabilidad e incertidumbre vinculadas con experiencias personales y son difíciles de modificar (Fernández Nistal, Pérez Ibarra, Peña Boone y Mercado Ibarra, 2011, p. 572). Las teorías implícitas, en la definición de Hernández Rojas (2012, p. 44), son configuraciones sobre un cierto dominio de la realidad, producto de experiencias personales y de conocimiento culturalmente compartido, "de naturaleza implícita, en el sentido de no ser completamente conscientes", que mantienen cierta coherencia interna, influyen en las acciones y son resistentes al cambio.
} 
conocida división dicotómica entre transformar el conocimiento y decir el conocimiento. El primer término remite al modelo de composición que corresponde a una producción experta, escribir para descubrir -la comprensión de lo que se intenta escribir cambia y crece durante el proceso de composición y el desarrollo conceptual y lingüístico se atribuye al efecto de la interacción entre el contenido y los aspectos retóricos (objetivos discursivos, género y audiencia)-. El segundo es un modelo inmaduro de producción por el cual se escribe de manera lineal -del contenido al espacio retórico- aquello que "se sabe" sobre el tema; se trata de un nivel de procesamiento superficial y con un mínimo compromiso.

Levin y Wagner (2006), mediante una tarea de escritura reflexiva, analizan las metáforas que emplean estudiantes de escuela secundaria para referirse a la escritura. Señalan que prefieren esta técnica menos directa y explícita por las dificultades del alumno para verbalizar su pensamiento metacognitivo, agravadas por el carácter complejo y multidimensional de la escritura. Los resultados evidencian que las metáforas refieren a dos visiones antagónicas de la escritura: como espacio cerrado y como espacio abierto. Desde la primera se la concibe como un proceso mecánico relativamente rígido -un conjunto de reglas y de expectativas respecto del contenido, el lector y su conducta escritural-, que enfatiza la linealidad y no implica actividad metacognitiva. Por el contrario, el espacio abierto es un sistema dinámico de procesos adaptativos e interpretativos, caracterizado por el pensamiento reflexivo y crítico, el control de la escritura hacia la auto-organización de nuevas formas construidas por procesos más sistemáticos y otros más creativos, que apoya un sentido de propiedad y autonomía. Asocian las concepciones de escritura que vehiculizan las metáforas con la visión trasmisiva vs. constructivista del aprendizaje y la enseñanza.

\subsection{Enfoque de la teoría social del aprendizaje}

White y Bruning (2005) indagan las creencias trasmisivas vs. transaccionales en estudiantes universitarios. El alumno con creencias predominantemente trasmisivas (alta trasmisión-baja transacción) conceptualiza la escritura como un medio de transferencia de información desde el autor al lector y demuestra escaso compromiso afectivo y cognitivo durante el proceso de composición. En cambio, el escritor con creencias predominantemente transaccionales (alta transacción-baja trasmisión) la concibe como una construcción activa, personal y crítica de un texto, que exige considerables niveles de implicación. Los resultados indican que los estudiantes con baja transacción obtienen puntuaciones inferiores en la 
calidad general del texto, mientras que aquellos que evidencian transacción alta logran mejor desarrollo temático, organización, fluidez, voz y calidad global del texto producido.

Hernández Rojas (2012), mediante entrevista y la técnica de pensar en voz alta, analiza las teorías implícitas de escritura, o estructuración de las conceptualizaciones como "sistemas constelados", de estudiantes de Química y Letras. Los resultados muestran diferencias en el tipo prevalente de teoría implícita de la escritura entre los alumnos de cada comunidad académica: preconstructiva en los primeros porque intentan realizar "una escritura transformadora sin conseguirlo del todo, aunque de ninguna manera es una escritura puramente receptivo-reproductiva"; constructiva en la mayoría de los segundos, una conceptualización "que aprecia la complejidad de la tarea compositiva” (Hernández Rojas, 2012, p. 56).

En esta línea de las concepciones como teorías implícitas, también se inscriben los trabajos de investigadores españoles: Mateos et al. (2011), Villalón Molina y Mateos (2009), Villalón Molina (2010). Esta última autora estudia las concepciones epistémica vs. reproductiva en educación media y superior y halla que, aunque los universitarios poseen una conceptualización más elaborada que los alumnos de secundaria, ambos están lejos de alcanzar una concepción epistémica de la escritura.

En la siguiente tabla (Tabla 1) se sintetizan los desarrollos previos en orden cronológico: 
Tabla 1

Concepciones de escritura y diversos sistemas de categorías

\begin{tabular}{|c|c|c|c|c|}
\hline Autores & \multicolumn{4}{|c|}{ Sistema de categorías } \\
\hline Hounsell (1984) & Como argumento & Como p & to de vista & Como arreglo, orden \\
\hline Biggs (1988) & \multicolumn{2}{|c|}{ Profunda } & \multicolumn{2}{|c|}{ Superficial } \\
\hline $\begin{array}{ll}\text { Scardamalia } & \text { y } \\
\text { Bereiter (1992) }\end{array}$ & \multicolumn{2}{|c|}{ Transformar el conocimiento } & \multicolumn{2}{|c|}{ Decir el conocimiento } \\
\hline $\begin{array}{l}\text { Campbell, Smith } \\
\text { y Brooker (1998) }\end{array}$ & \multicolumn{2}{|c|}{ Contructivista } & \multicolumn{2}{|c|}{ Reproductiva } \\
\hline $\begin{array}{l}\text { Lavelle }{ }^{y} \\
\text { Zuercher, (2001) }\end{array}$ & \multicolumn{2}{|c|}{$\begin{array}{c}\text { Profunda } \\
\text { (Elaboración y Reflexión-revisión) }\end{array}$} & \multicolumn{2}{|c|}{$\begin{array}{c}\text { Superficial } \\
\text { (Baja autoeficacia, Espontáneo-intuitivo y } \\
\text { Procedural) }\end{array}$} \\
\hline $\begin{array}{l}\text { White y Bruning } \\
\text { (2005) }\end{array}$ & \multicolumn{2}{|c|}{ Transaccional } & \multicolumn{2}{|c|}{ Trasmisiva } \\
\hline $\begin{array}{l}\text { Levin y Wagner } \\
\text { (2006) }\end{array}$ & \multicolumn{2}{|c|}{ Espacio abierto } & \multicolumn{2}{|c|}{ Espacio cerrado } \\
\hline $\begin{array}{l}\text { Lavelle } y \\
\text { Bushrow (2007) }\end{array}$ & \multicolumn{2}{|c|}{$\begin{array}{c}\text { Profunda } \\
\text { (Intutitivo, Elaborativo y Científico) }\end{array}$} & \multicolumn{2}{|c|}{$\begin{array}{c}\text { Superficial } \\
\text { (No revisión, Baja autoeficacia, Orientado a } \\
\text { la tarea y Escultor) }\end{array}$} \\
\hline $\begin{array}{l}\text { Ellis, Taylor y } \\
\text { Drury (2007) }\end{array}$ & \multicolumn{2}{|c|}{ Cohesiva } & \multicolumn{2}{|c|}{ Fragmentada } \\
\hline $\begin{array}{l}\text { Bóscolo, Arfé y } \\
\text { Quarisa (2007) }\end{array}$ & Elaboración y revisi & \multicolumn{2}{|c|}{ Punto de vista persona } & $\begin{array}{l}\text { Trasmisión del } \\
\text { conocimiento }\end{array}$ \\
\hline $\begin{array}{l}\text { Villalón Molina } \\
\text { (2010) }\end{array}$ & \multicolumn{2}{|c|}{ Epistémica } & \multicolumn{2}{|c|}{ Reproductiva } \\
\hline $\begin{array}{l}\text { Hernández Rojas } \\
\text { (2012) }\end{array}$ & \multicolumn{2}{|c|}{ Constructiva } & \multicolumn{2}{|c|}{ Reproductiva } \\
\hline
\end{tabular}

Fuente: de la autora.

Como queda manifiesto, aunque con variaciones terminológicas, la mayoría de los estudios enfatizan los dos enfoques que distinguiera Biggs (1988), profundo vs. superficial, los que en nuestro caso, en seguimiento de Lonka (1996), designamos elaboración vs. reproducción:

1) Escritura como elaboración, perspectiva que, por un lado, aprovecha el potencial epistémico de la actividad escritural (Wells, 1990) ${ }^{6}$ (ej. de ítem de nuestra escala: "La escritura es un proceso reflexivo que me permite una mayor comprensión de lo que pienso") y, por otro, lleva al escritor a decidir cómo organizar el contenido de su producción teniendo en cuenta los aspectos retóricos. Un texto bien elaborado exige un esfuerzo de adecuación al lector: de relieve, de elección de los términos, de

\footnotetext{
${ }^{6}$ La producción escrita es un proceso cognitivo y epistémico de elaboración del conocimiento y de reelaboración del pensamiento (Serrano de Moreno, 2001, p. 26), esto es, no es sólo una herramienta de comunicación de "lo que se sabe" o "lo que se juzga" respecto de alguna temática, sino un instrumento para objetivar, organizar, revisar, modificar, acrecentar, clarificar el pensamiento y la reflexión.
}

Volumen 13, Número 3, Año 2013, ISSN 1409-4703 
organización de las ideas (ej.: "Mientras escribo, tengo en cuenta la comprensión de mi trabajo por parte del lector"). Implica la producción de un plan comprehensivo tanto por el número de núcleos conceptuales que incorpora como por su interconexión, la que el escritor emplea durante todo el proceso como "instrumento de monitoreo" (Vázquez y Jacob, 2006, p. 59). Exige estrategias complejas -atender a los aspectos globales del texto: macroestructurales, superestructurales y del modelo de situación, en la terminología de van Dijk y Kintsch (1983)-, reflexión, autonomía, un tratamiento profundo de la información ${ }^{7}$ y la revisión como actividad central para evaluar el significado y su estructura, que implica una cierta sofisticación metacognitiva.

2) Escritura como reproducción, que involucra ausencia de planeamiento, selección de la información paso a paso sin una representación global del texto, una estrategia de listado por lo que el escrito adopta la forma de una colección débil de ideas, sigue un modelo de tipo secuencial (inclusión de información de manera yuxtapuesta y acumulativa, con instancias del método empírico de copia ${ }^{8}$ ) y lineal -del contenido al espacio retórico (ej.: "En la composición de un texto académico, se lee para buscar información y se escribe para mostrar el conocimiento adquirido")-; como resultado, lo que emerge es el texto final, quizás con alguna revisión menor a nivel léxico o gramatical $^{9}$, el agregado de una oración aquí o allá (la atención se pone en el nivel superficial del texto). Como señalan Silva y Nicholls (1993), este enfoque no correlaciona con la competencia escritural ni con el compromiso (ej.: "Lo más importante en la escritura es respetar las reglas de gramática, puntuación y organización").

${ }^{7}$ La información y su organización están subordinadas a la posición global interpretativa elegida por el escritor; siguen un modelo recursivo -el contenido temático se interrelaciona con el espacio retórico, lo cual lleva a reelaborarlo y a transformarlo para adecuarse a la situación, luego, el texto en sí mismo agrega al espacio de contenido-.

${ }^{8}$ Alda Alves (1992) señala que el autor parece un "ventrílocuo" porque sólo habla por boca de otros, ya sea citándolos literalmente, ya sea parafraseando sus ideas. Este estilo es fácilmente reconocible: los párrafos se suceden alternando expresiones como "Para Fulano", "Según Sultano", "Como afirma Perengano", "Como Sultano observa", hasta agotar el correspondiente conjunto de verbos sinónimos o de significado bastante cercano -decir, expresar, afirmar, postular, manifestar, opinar, mostrar, explicar, etc.-.

${ }^{9}$ Keith Hjortshoj (2001; citada por Carlino, 2004) señala que "desde la perspectiva del que escribe, todo texto 'fragua' en algún punto del proceso, endureciéndose como cemento. Más allá de ese punto, cuando lenguaje y pensamiento han perdido maleabilidad, se requiere algo del orden de la dinamita para promover cambios profundos. Los escritores más experimentados dilatan este punto de solidificación, reconsideran y revisan a fondo su trabajo antes de dar por terminado un primer borrador completo. La escritura de los estudiantes, en cambio, tiende a fraguar casi en el momento en que toca el papel (...). Los primeros pensamientos se vuelven los últimos, y los segundos pensamientos parecen destructivos". (p. 324) 


\section{Metodología}

\subsection{Instrumento}

La escala Concepciones de Escritura Académica en el Doctorado se conforma sobre la base del Inventario de Escritura Académica en el Posgrado de 100 ítems, que produjéramos a partir de la traducción al Español de ítems procedentes de instrumentos estandarizados, los que adaptáramos para ajustarlos a nuestra dimensionalización teórica de la variable y para contextualizarlos en la producción de una Tesis doctoral, más algunos ítems generados ad hoc (cfr. Difabio de Anglat, 2012, presenta una versión abreviada de dicho inventario).

Está compuesta por 33 ítems -en cinco opciones de respuesta, desde muy de acuerdo a muy en desacuerdo-: 2 tomados de Inventory of Graduate Writing Processes (Lavelle y Bushrow, 2007), 12 adaptados de Inventory of Processes in College Composition (Lavelle y Guarino, 2003), 5 de Writing Self-Regulatory Efficacy Scale (Zimmerman y Bandura, 1994) y también 5 de Writing Process Questionnaire (Lonka, 1996); los restantes 9, elaborados ad hoc. En la siguiente tabla (ver Tabla 2), se los distribuyen desde las tres categorías de la escritura -Naturaleza y funciones, Planificación y textualización, Revisión- que distinguiéramos en seguimiento de Villalón Molina (2010):

Tabla 2

\section{Concepciones de escritura académica por categorías}

\begin{tabular}{|c|c|c|}
\hline & Escritura como elaboración & Escritura como reproducción \\
\hline & $\begin{array}{l}\text { La escritura es un proceso reflexivo que me } \\
\text { permite una mejor comprensión de lo que } \\
\text { pienso. } \\
\text { La escritura desarrolla las habilidades } \\
\text { intelectuales. } \\
\text { La escritura me ayuda a organizar la } \\
\text { información en mi mente. } \\
\text { Mientras escribo la Tesis, sigo una } \\
\text { secuencia de planificación, redacción, } \\
\text { revisión para cada capítulo. }\end{array}$ & $\begin{array}{l}\text { La escritura le informa al lector aquello que } \\
\text { sabe el autor. } \\
\text { En la composición de un texto académico, se } \\
\text { lee para buscar información y se escribe para } \\
\text { mostrar el conocimiento adquirido. } \\
\text { El texto académico se organiza como un } \\
\text { repertorio de tópicos que se desarrollan } \\
\text { secuencialmente de modo ordenado. } \\
\text { El texto académico debe ser impersonal, } \\
\text { aséptico, porque su principal finalidad es } \\
\text { informativa. }\end{array}$ \\
\hline & $\begin{array}{l}\text { Por lo general, examino con sumo cuidado } \\
\text { los requisitos del tipo de escrito que debo } \\
\text { producir. } \\
\text { Tiendo a invertir algún tiempo en reflexionar } \\
\text { sobre la tarea de escritura antes de } \\
\text { comenzarla. } \\
\text { Para la producción de la Tesis, examino } \\
\text { exhaustivamente la investigación previa. } \\
\text { Comienzo a escribir con algún plan de } \\
\text { escritura (de mayor o menor detalle). }\end{array}$ & $\begin{array}{l}\text { Me gusta que las tareas de escritura que me } \\
\text { asignan, tengan pautas claras con todos los } \\
\text { detalles. } \\
\text { Por lo general, para escribir un buen marco } \\
\text { teórico alcanza con la lectura de un número } \\
\text { delimitado de obras importantes. } \\
\text { Si se aconseja que una sección de la Tesis } \\
\text { tenga un número determinado de hojas, trato } \\
\text { de producir esa cifra. } \\
\text { Una estrategia eficiente para producir un }\end{array}$ \\
\hline
\end{tabular}




\begin{tabular}{|c|c|c|}
\hline $\begin{array}{l}\text { Planifica- } \\
\text { ción y } \\
\text { textualiza- } \\
\text { ción }\end{array}$ & $\begin{array}{l}\text { Trato de elaborar buenos esquemas para } \\
\text { las secciones principales de un capítulo. } \\
\text { Localizo y empleo fuentes de referencia } \\
\text { cuando necesito documentar un punto } \\
\text { importante. } \\
\text { Comparo y contrasto ideas para hacer mi } \\
\text { texto más claro. } \\
\text { Mientras escribo, tengo en cuenta la } \\
\text { comprensión de mi trabajo por parte del } \\
\text { lector. } \\
\text { Pienso sobre cómo me está quedando el } \\
\text { escrito. } \\
\text { Trato de que mi escritura satisfaga los } \\
\text { estándares de calificación de un evaluador } \\
\text { exigente. }\end{array}$ & $\begin{array}{l}\text { buen texto académico es elegir a un autor } \\
\text { reconocido y seguir la estructura de su obra. } \\
\text { Empleo muchas definiciones textuales para } \\
\text { hacer mi escrito más claro. } \\
\text { Los profesores son la audiencia más } \\
\text { importante. } \\
\text { Mi intención cuando escribo es simplemente } \\
\text { contestar la pregunta que me formulé. } \\
\text { Lo más importante en la escritura es respetar } \\
\text { las reglas de gramática, puntuación y } \\
\text { organización. } \\
\text { Si estudiara gramática y puntuación, mi } \\
\text { escritura mejoraría mucho. }\end{array}$ \\
\hline Revisión & $\begin{array}{l}\text { Cuando he escrito un texto largo, me } \\
\text { esfuerzo por encontrar y corregir todos mis } \\
\text { errores gramaticales y de estilo. } \\
\text { Reviso el borrador para hacerlo más } \\
\text { organizado. } \\
\text { Con frecuencia reviso mi escrito varias } \\
\text { veces antes de considerarlo terminado. }\end{array}$ & $\begin{array}{l}\text { No tiene sentido mostrarle el escrito a } \\
\text { persona alguna hasta tanto esté finalizado. } \\
\text { Por lo general, elaboro los trabajos escritos a } \\
\text { último momento y, a pesar de ello, me suele ir } \\
\text { bien. } \\
\text { Con frecuencia, reviso una sola vez y al } \\
\text { finalizar el texto }\end{array}$ \\
\hline
\end{tabular}

Fuente: de la autora.

\subsection{Muestra}

Se conforma de 39 doctorandos en Educación de Argentina (de la Universidad Nacional de Cuyo, Mendoza, y de la Universidad Católica de Cuyo, sedes San Juan y San Luis), 35 mujeres y 4 varones, a los que se les aplica el inventario en forma autoadministrada vía correo electrónico, previo consentimiento del alumno para recibirlo; se puntúa cada ítem desde 4 (muy de acuerdo) a 0 (muy en desacuerdo).

\subsection{Análisis}

Partimos de estimar los siguientes índices descriptivos por ítem: promedio, desviación estándar (D.E.), porcentajes de respuesta por opción y proporción de estudiantes por categorías de escritores según Elaboración y Reproducción. Luego, ponderamos la confiabilidad de cada subescala a través del alfa de Cronbach. En tercer lugar, evaluamos la potencialidad discriminativa de los ítems por el contraste entre grupos extremos $-30 \%$ superior y 30\% inferior- del porcentaje de respuestas superiores (puntajes $4 \circ 3$ ), llamado análisis de los ítems de los tercios superior e inferior (Grant, 2002). Finalmente, calculamos las correlaciones -rho de Spearman- entre las subescalas y también con la productividad 
(número de horas dedicadas a la escritura por semana y número de hojas efectivamente producidas).

\section{Resultados}

\subsection{Descriptivos}

Las tablas 3 y 4, por ítem, presentan la Media, la Desviación estándar (D.E.) y los porcentajes de respuestas en cada alternativa de las subescalas Escritura como elaboración y Escritura como reproducción, respectivamente.

Tabla 3

Escritura como elaboración

\begin{tabular}{|c|c|c|c|c|c|c|c|}
\hline \multirow[t]{2}{*}{ Ítems } & \multirow[t]{2}{*}{ Media } & \multirow[t]{2}{*}{ D.E. } & \multicolumn{5}{|c|}{ \% de respuesta por opción } \\
\hline & & & 4 & 3 & 2 & 1 & 0 \\
\hline $\begin{array}{l}\text {... proceso reflexivo que me permite una mayor } \\
\text { comprensión de lo que pienso. }\end{array}$ & 3,15 & ,779 & 36 & 46 & 15 & 3 & 0 \\
\hline ... desarrolla las habilidades intelectuales. & 3,28 & ,826 & 46 & 41 & 8 & 5 & 0 \\
\hline $\begin{array}{l}\text {.. me ayuda a organizar la información en mi } \\
\text { mente. }\end{array}$ & 3,41 & ,909 & 64 & 18 & 13 & 5 & 0 \\
\hline $\begin{array}{l}\text {.. sigo una secuencia de planificación, } \\
\text { textualización y revisión para cada capítulo. }\end{array}$ & 3,03 & 810 & 26 & 56 & 15 & 0 & 3 \\
\hline $\begin{array}{l}\text {... examino con cuidado los requisitos del tipo de } \\
\text { texto que debo producir. }\end{array}$ & 3,21 & ,923 & 43 & 41 & 10 & 3 & 3 \\
\hline $\begin{array}{l}\text {... reflexionar sobre la tarea de escritura antes de } \\
\text { comenzarla. }\end{array}$ & 3,00 & 827 & 26 & 56 & 10 & 8 & 0 \\
\hline $\begin{array}{l}\text {.. examino exhaustivamente la investigación } \\
\text { previa. }\end{array}$ & 2,10 & 995, & 5 & 36 & 26 & 30 & 3 \\
\hline $\begin{array}{l}\text {... algún plan de escritura (de mayor o menor } \\
\text { detalle). }\end{array}$ & 2,95 & 944 & 25 & 56 & 8 & 8 & 3 \\
\hline $\begin{array}{l}\text { Trato de elaborar buenos esquemas para las } \\
\text { secciones principales de un capítulo. }\end{array}$ & 3,13 & 656 & 28 & 57 & 15 & 0 & 0 \\
\hline $\begin{array}{l}\text {.. fuentes de referencia cuando necesito } \\
\text { documentar un punto importante. }\end{array}$ & 3,38 & 847 & 56 & 31 & 8 & 5 & 0 \\
\hline $\begin{array}{l}\text { Comparo y contrasto ideas para hacer mi texto } \\
\text { más claro. }\end{array}$ & 3,08 & 957 & 36 & 46 & 10 & 5 & 3 \\
\hline $\begin{array}{l}\ldots \text { tengo en cuenta la comprensión de mi trabajo } \\
\text { por parte del lector. }\end{array}$ & 3,18 & 914 & 41 & 43 & 10 & 3 & 3 \\
\hline Pienso sobre cómo me está quedando el escrito. & 3,31 & ,799 & 46 & 44 & 5 & 5 & 0 \\
\hline $\begin{array}{l}\ldots \text { mi escritura satisfaga los estándares de } \\
\text { calificación de un evaluador exigente. }\end{array}$ & 3,28 & ,793 & 46 & 38 & 13 & 3 & 0 \\
\hline $\begin{array}{l}\text {... me esfuerzo por encontrar y corregir todos mis } \\
\text { errores gramaticales y de estilo. }\end{array}$ & 3,05 & 972 & 33 & 43 & 18 & 3 & 3 \\
\hline Reviso el borrador ... más organizado & 3,02 & ,932 & 36 & 43 & 13 & 5 & 3 \\
\hline $\begin{array}{l}\text { Con frecuencia reviso mi escrito varias veces antes } \\
\text { de considerarlo terminado. }\end{array}$ & 3,26 & ,938 & 51 & 28 & 18 & 0 & 3 \\
\hline
\end{tabular}

Fuente: elaboración propia de la autora. 
A excepción del séptimo ítem ("Para la producción de la Tesis, examino exhaustivamente la investigación previa"), felizmente la sumatoria de las opciones "muy de acuerdo" y "de acuerdo" supera el 75\%. El porcentaje mayor (90\%) corresponde al decimotercero (“Pienso sobre cómo me está quedando el escrito").

Tabla 4

Escritura como reproducción

\begin{tabular}{|c|c|c|c|c|c|c|c|}
\hline \multirow[t]{2}{*}{ Ítems } & \multirow[t]{2}{*}{ Media } & \multirow[t]{2}{*}{ D.E. } & \multicolumn{5}{|c|}{$\%$ de respuesta por opción } \\
\hline & & & 4 & 3 & 2 & 1 & $\mathbf{0}$ \\
\hline ... informa al lector aquello que sabe el autor. & 3,15 & 933 & 43 & 36 & 13 & 8 & 0 \\
\hline $\begin{array}{l}\text {... se lee para buscar información y se escribe para } \\
\text { mostrar el conocimiento adquirido. }\end{array}$ & 2,72 & 1,025 & 23 & 44 & 15 & 18 & 0 \\
\hline $\begin{array}{l}\text {... repertorio de tópicos que se desarrollan } \\
\text { secuencialmente de modo ordenado. }\end{array}$ & 2,97 & ,903 & 28 & 48 & 18 & 3 & 3 \\
\hline $\begin{array}{l}\ldots \text { debe ser impersonal, aséptico, porque su } \\
\text { principal finalidad es informativa. }\end{array}$ & 1,97 & 1,287 & 13 & 28 & 15 & 31 & 13 \\
\hline $\begin{array}{l}\text {... las tareas de escritura que me asignan, tengan } \\
\text { pautas claras con todos los detalles. }\end{array}$ & 3,26 & ,818 & 46 & 36 & 15 & 3 & 0 \\
\hline $\begin{array}{l}\text {... para escribir un buen marco teórico alcanza con } \\
\text { la lectura de un número delimitado de obras } \\
\text { importantes. }\end{array}$ & 2,13 & 1,281 & 15 & 31 & 16 & 28 & 10 \\
\hline $\begin{array}{l}\text {... número determinado de hojas, trato de producir } \\
\text { esa cifra. }\end{array}$ & 3,05 & ,724 & 28 & 49 & 23 & 0 & 0 \\
\hline $\begin{array}{l}\text {... elegir a un autor reconocido y seguir la } \\
\text { estructura de su obra. }\end{array}$ & 1,82 & 1,167 & 10 & 20 & 18 & 44 & 8 \\
\hline $\begin{array}{l}\text { Empleo muchas definiciones textuales para hacer } \\
\text { más claro mi escrito. }\end{array}$ & 2,54 & 1,072 & 20 & 36 & 21 & 23 & 0 \\
\hline Los profesores son la audiencia más importante. & 2,56 & 1,071 & 18 & 43 & 18 & 18 & 3 \\
\hline $\begin{array}{l}\text { Mi intención cuando escribo es simplemente } \\
\text { contestar la pregunta que me formulé. }\end{array}$ & 1,62 & 1,042 & 8 & 10 & 25 & 49 & 8 \\
\hline $\begin{array}{l}\text { Lo más importante ... respetar las reglas de } \\
\text { gramática, puntuación y organización. }\end{array}$ & 2,67 & 1,199 & 28 & 38 & 8 & 23 & 3 \\
\hline $\begin{array}{l}\text { Si estudiara gramática y puntuación, mi escritura } \\
\text { mejoraría mucho. }\end{array}$ & 3,05 & ,826 & 31 & 49 & 15 & 5 & 0 \\
\hline $\begin{array}{l}\text { No tiene sentido mostrarle el escrito a persona } \\
\text { alguna hasta tanto esté finalizado. }\end{array}$ & 1,03 & 1,224 & 8 & 8 & 5 & 38 & 41 \\
\hline $\begin{array}{l}\ldots \text { elaboro los trabajos escritos a último momento } \\
\text { y, a pesar de ello, me suele ir bien. }\end{array}$ & 1,21 & 1,341 & 8 & 5 & 23 & 44 & 20 \\
\hline ... reviso una sola vez y al finalizar el texto. & 1,36 & 1,112 & 13 & 5 & 8 & 38 & 36 \\
\hline
\end{tabular}

Fuente: elaboración propia de la autora.

En esta subescala, la sumatoria de las opciones "muy de acuerdo" y "de acuerdo" muestra una gran variabilidad: cuatro ítems no superan el 18\% ("Mi intención... es simplemente contestar la pregunta que me formulé", "No tiene sentido mostrarle el escrito a persona alguna hasta tanto esté finalizado", "Por lo general, elaboro los trabajos escritos Volumen 13, Número 3, Año 2013, ISSN 1409-4703 
solicitados a último momento..." -este último obtiene 13\%, el porcentaje menor de toda la muestra-, y "Con frecuencia, reviso una sola vez y al finalizar el texto"); tres se inscriben en el rango $30 \%-46 \%$ ("Una estrategia eficiente... es elegir a un autor reconocido y seguir la estructura de su obra", "El texto académico debe ser impersonal, aséptico..." y “... para escribir un buen marco teórico alcanza con la lectura de un número delimitado de obras importantes"); cuatro, entre 56\% y 67\% (“Empleo muchas definiciones textuales...", "Los profesores son la audiencia más importante", "Lo más importante en la escritura es respetar las reglas de gramática, puntuación y organización" y "En la composición de un texto académico, se lee para buscar información y se escribe para mostrar el conocimiento adquirido"); finalmente, cinco superan el 76\% (“El texto académico se organiza como un repertorio de tópicos...", "Si se aconseja que una sección de la Tesis tenga un número determinado de hojas, trato de producir esa cifra", "La escritura le informa al lector aquello que sabe el autor", "Si estudiara gramática y puntuación, mi escritura mejoraría mucho" y "Me gusta que las tareas de escritura que me asignan, tengan pautas claras con todos los detalles" -el que obtiene $82 \%$, el porcentaje mayor de esta subescala-). En suma, los indicadores de la falta de revisión son los que evidencian menor acuerdo y los correspondientes a la conceptualización de la naturaleza y funciones de la escritura como trasmisión del conocimiento, los que manifiestan el mayor acuerdo.

Como en White y Bruning (2005, p. 177), conformamos cuatro categorías de escritores (alta elaboración-alta reproducción; alta elaboración-baja reproducción; baja elaboración-alta reproducción; baja elaboración-baja reproducción) desde el 25\% superior e inferior del puntaje total en Elaboración y en Reproducción. Los porcentajes hallados en la muestra se grafican a continuación (ver Gráfico 1): 


\section{Gráfico 1}

Categorías de escritores según Elaboración y Reproducción

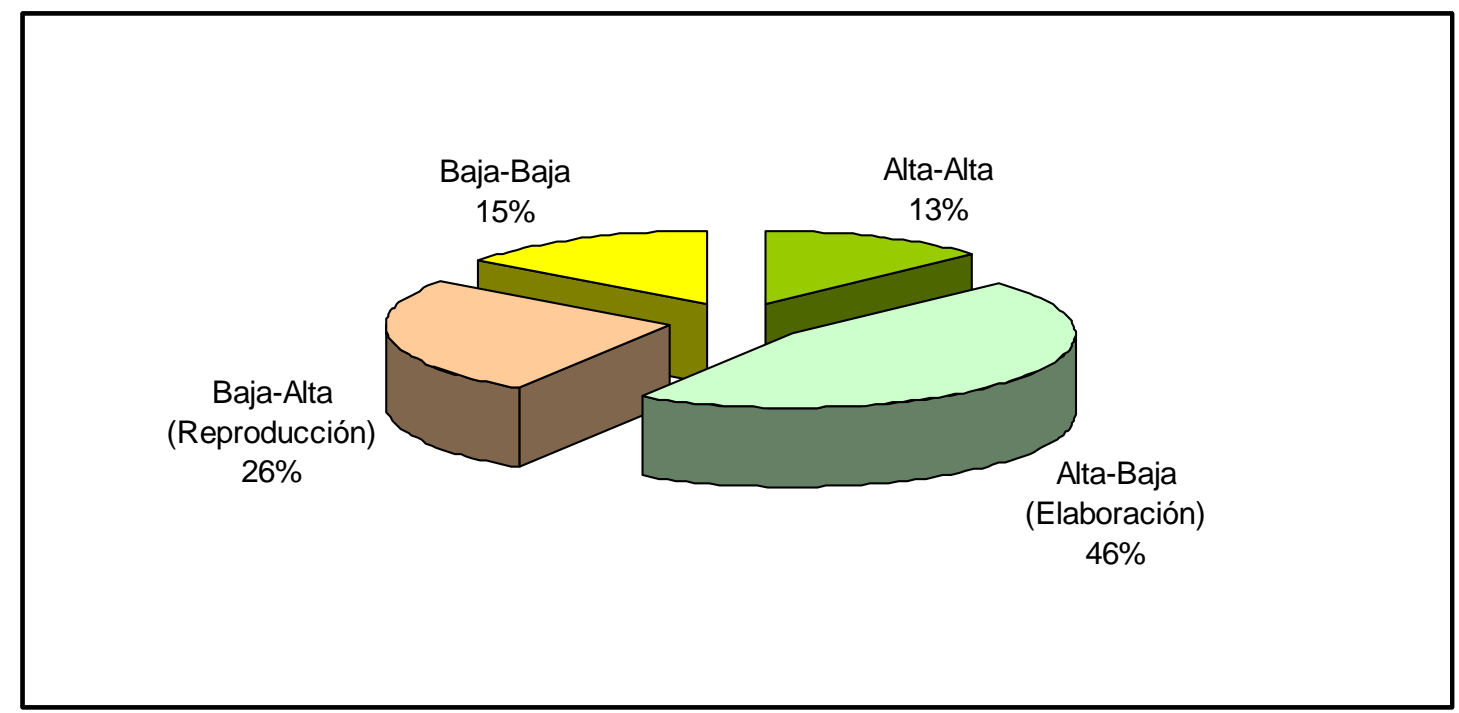

Aunque los estudiantes con concepciones elaborativas superan a quienes manifiestan concepciones reproductivas, el porcentaje de estos últimos es considerable (41\%) cuando se suma también la categoría baja elaboración-baja reproducción.

\subsection{Análisis de confiabilidad}

Los coeficientes de confiabilidad por variable son (Tabla 5):

Tabla 5

Confiabilidad por escala

\begin{tabular}{lc}
\hline \multicolumn{1}{c}{ Concepciones } & $\alpha$ de Cronbach \\
\hline Escritura como Elaboración & 0,93 \\
Escritura como Reproducción & 0,91 \\
\hline
\end{tabular}

Los índices significan muy buena confiabilidad, ya que una aplicación del instrumento nos informa más del $80 \%$ de lo que necesitamos saber.

\subsection{Análisis de la potencialidad discriminativa de los ítems}

Calculamos la discriminación de los ítems mediante un procedimiento sencillo: ordenar los resultados en la muestra total en forma decreciente; computar el porcentaje de respuestas superiores (3 ó 4) por ítem en el 30\% superior y en el 30\% inferior, porcentaje que permite formar grupos extremos bien diferentes (Grant, 2002); y hallar el "índice de Volumen 13, Número 3, Año 2013, ISSN 1409-4703 
discriminación" (ID), resultado de la resta del porcentaje entre el grupo superior (GS) y el inferior (Gl). Los resultados son (ver tablas 6 y 7 ):

Tabla 6

Poder discriminante de los ítems en escritura como elaboración

\begin{tabular}{|c|c|c|c|}
\hline & \multicolumn{2}{|c|}{ Grupos } & \\
\hline Ítems & Sup. & Inf. & ID \\
\hline $\begin{array}{l}\text { La escritura es un proceso reflexivo que me permite una mayor comprensión } \\
\text { de lo que pienso. }\end{array}$ & $92 \%$ & $58 \%$ & $34 \%$ \\
\hline La escritura desarrolla las habilidades intelectuales. & $100 \%$ & $67 \%$ & $33 \%$ \\
\hline La escritura me ayuda a organizar la información en mi mente. & $100 \%$ & $50 \%$ & $50 \%$ \\
\hline $\begin{array}{l}\text { Mientras escribo la Tesis, sigo una secuencia de planificación, textualización y } \\
\text { revisión para cada capítulo. }\end{array}$ & $100 \%$ & $58 \%$ & $42 \%$ \\
\hline $\begin{array}{l}\text { Por lo general, examino con cuidado los requisitos del tipo de texto que debo } \\
\text { producir. }\end{array}$ & $100 \%$ & $59 \%$ & $41 \%$ \\
\hline $\begin{array}{l}\text { Tiendo a invertir algún tiempo en reflexionar sobre la tarea de escritura antes } \\
\text { de comenzarla. }\end{array}$ & $100 \%$ & $67 \%$ & $33 \%$ \\
\hline $\begin{array}{l}\text { Para la producción de la Tesis, examino exhaustivamente la investigación } \\
\text { previa. }\end{array}$ & $59 \%$ & $8 \%$ & $51 \%$ \\
\hline Comienzo a escribir con algún plan de escritura ... & $100 \%$ & $50 \%$ & $50 \%$ \\
\hline $\begin{array}{l}\text { Trato de elaborar buenos esquemas para las secciones principales de mi } \\
\text { informe. }\end{array}$ & $100 \%$ & $67 \%$ & $33 \%$ \\
\hline $\begin{array}{l}\text { Localizo y empleo fuentes de referencia cuando necesito documentar un punto } \\
\text { importante. }\end{array}$ & $100 \%$ & $67 \%$ & $33 \%$ \\
\hline Comparo y contrasto ideas para hacer mi texto más claro. & $92 \%$ & $50 \%$ & $42 \%$ \\
\hline $\begin{array}{l}\text { Mientras escribo, tengo en cuenta la comprensión de mi trabajo por parte del } \\
\text { lector. }\end{array}$ & $100 \%$ & $58 \%$ & $42 \%$ \\
\hline Pienso sobre cómo me está quedando el escrito. & $100 \%$ & $67 \%$ & $33 \%$ \\
\hline $\begin{array}{l}\text { Trato de que mi escritura satisfaga los estándares de calificación de un } \\
\text { evaluador exigente. }\end{array}$ & $100 \%$ & $58 \%$ & $42 \%$ \\
\hline $\begin{array}{l}\text { Cuando he escrito un texto largo, me esfuerzo por encontrar y corregir todos } \\
\text { mis errores gramaticales y de estilo. }\end{array}$ & $100 \%$ & $33 \%$ & $67 \%$ \\
\hline Reviso el borrador para hacerlo más organizado. & $100 \%$ & $42 \%$ & $58 \%$ \\
\hline Con frecuencia reviso mi escrito varias veces antes de considerarlo terminado. & $100 \%$ & $33 \%$ & $67 \%$ \\
\hline
\end{tabular}

Fuente: elaboración propia de la autora.

La totalidad de los ítems resulta discriminativa: seis ítems alcanzan discriminación buena (de 30 a 39\% de diferencia entre el grupo superior y el inferior) y once, muy buena (40\% o más); por otra parte, en esta última dos ítems evidencian índices mayores al $60 \%$ de diferencia entre el grupo superior y el inferior, esto es, son indicadores con muy alto poder discriminante. 
Tabla 7

Poder discriminante de los ítems en escritura como reproducción

\begin{tabular}{|c|c|c|c|}
\hline & Gru & & \\
\hline İtems & Sup. & Inf. & ID \\
\hline La escritura le informa al lector aquello que sabe el autor. & $100 \%$ & $42 \%$ & $58 \%$ \\
\hline $\begin{array}{l}\text { En la composición de un texto académico, se lee para buscar información y se } \\
\text { escribe para mostrar el conocimiento adquirido. }\end{array}$ & $100 \%$ & $25 \%$ & $75 \%$ \\
\hline $\begin{array}{l}\text { El texto académico se organiza como un repertorio de tópicos que se } \\
\text { desarrollan secuencialmente de modo ordenado. }\end{array}$ & $100 \%$ & $33 \%$ & $67 \%$ \\
\hline $\begin{array}{l}\text { El texto académico debe ser impersonal, aséptico, porque su principal finalidad } \\
\text { es informativa. }\end{array}$ & $92 \%$ & $0 \%$ & $92 \%$ \\
\hline $\begin{array}{l}\text { Me gusta que las tareas de escritura que me asignan, tengan pautas claras con } \\
\text { todos los detalles. }\end{array}$ & $100 \%$ & $50 \%$ & $50 \%$ \\
\hline $\begin{array}{l}\text { Por lo general, para escribir un buen marco teórico alcanza con la lectura de un } \\
\text { número delimitado de obras importantes. }\end{array}$ & $92 \%$ & $0 \%$ & $92 \%$ \\
\hline $\begin{array}{l}\text { Si se aconseja que una sección de la Tesis tenga un número determinado de } \\
\text { hojas, trato de producir esa cifra. }\end{array}$ & $92 \%$ & $75 \%$ & $17 \%$ \\
\hline $\begin{array}{l}\text { Una estrategia eficiente para producir un buen texto académico es elegir a un } \\
\text { autor reconocido y seguir la estructura de su obra. }\end{array}$ & $75 \%$ & $0 \%$ & $75 \%$ \\
\hline Empleo muchas definiciones textuales para hacer más claro mi escrito. & $92 \%$ & $33 \%$ & $59 \%$ \\
\hline Los profesores son la audiencia más importante. & $92 \%$ & $16 \%$ & $76 \%$ \\
\hline $\begin{array}{l}\text { Mi intención cuando escribo es simplemente contestar la pregunta que me } \\
\text { formulé. }\end{array}$ & $50 \%$ & $0 \%$ & $50 \%$ \\
\hline $\begin{array}{l}\text { Lo más importante en la escritura es respetar las reglas de gramática, } \\
\text { puntuación y organización. }\end{array}$ & $92 \%$ & $42 \%$ & $50 \%$ \\
\hline Si estudiara gramática y puntuación, mi escritura mejoraría mucho. & $83 \%$ & $75 \%$ & $8 \%$ \\
\hline $\begin{array}{l}\text { No tiene sentido mostrarle el escrito a persona alguna hasta tanto esté } \\
\text { finalizado. }\end{array}$ & $50 \%$ & $0 \%$ & $50 \%$ \\
\hline $\begin{array}{l}\text { Por lo general, elaboro los trabajos escritos solicitados a último momento y, a } \\
\text { pesar de ello, me suele ir bien. }\end{array}$ & $42 \%$ & $0 \%$ & $42 \%$ \\
\hline Con frecuencia, reviso una sola vez y al finalizar el texto. & $58 \%$ & $0 \%$ & $58 \%$ \\
\hline
\end{tabular}

Fuente: elaboración propia de la autora.

El $87,5 \%$ de la escala resulta discriminativo: catorce ítems a nivel muy bueno, mientras que seis (el $37,5 \%$ del instrumento) muestran discriminación alta.

\subsection{Análisis de las correlaciones}

Sólo se halla la correlación esperada entre la concepción de la escritura como elaboración y el número de horas dedicadas a la tarea por semana (rho =0,32, $p=0,05$ ); no así con el número de hojas efectivamente producidas ( $r h o=0,012, p=0,94$ ). Por otra parte, aunque sin significación estadística, ambas subescalas no están asociadas (rho = -0,27, $p=$ $0,10)$. 


\section{Conclusiones}

La aprehensión de las exigencias y estándares del discurso científico de nivel doctoral está fuertemente condicionada por las concepciones de escritura del estudiante, las que a su turno inciden en sus actividades escriturales. De allí que es imprescindible que los doctorandos sustituyan el enfoque superficial (en nuestra muestra, hallamos un porcentaje relativamente alto -el 41\%- de estudiantes en esta categoría) por aquel que les permitirá escribir su Tesis: un enfoque profundo, basado en una posición proactiva hacia la producción de un nuevo significado y el empleo de las estrategias conducentes (Lavelle y Bushrow, 2007).

A fin de implementar alguna forma de andamiaje que facilite dicho tránsito, se requiere evaluar los niveles de partida; para ello, es necesario disponer de un instrumento confiable y de fácil aplicación para que los datos obtenidos con él puedan usarse significativamente.

La escala sobre concepciones de escritura académica construida a partir de la combinación de ítems de cuatro instrumentos estandarizados y de algunos elaborados ad hoc, manifiesta, según el alfa de Cronbach, un índice de 0,93 para la subescala Escritura como elaboración y de 0,91 en el caso de Escritura como reproducción, coeficientes que la teoría psicométrica juzga considerables.

También, evidencia que resultan discriminativos todos los ítems de la primera subescala (a nivel bueno o muy bueno) y el $87,5 \%$ de los ítems de la segunda (a nivel muy bueno). Por otra parte, 8 de los 33 ítems del instrumento completo (el 24\%) alcanzan índices mayores al $60 \%$ de diferencia entre el grupo superior y el inferior, esto es, son indicadores con muy alto poder discriminante.

Sin embargo, el tamaño de nuestra muestra no admite el análisis factorial imprescindible para validar el instrumento. Nuevas investigaciones deberán efectuar el proceso de validación e incorporar la variable "calidad del texto producido" para profundizar el rol de las concepciones de escritura en la tarea compositiva de una Tesis doctoral.

\section{Referencias}

Alves, Alda. (1992). A "revisao da bibliografia" em teses e dissertaçoes: Meus tipos inesquecíveis. Cadernos de Pesquisa, 81, 53-60.

Biggs, John. (1988). Approaches to learning and essay writing. En Ronald Schmeck (Ed.), Learning strategies and learning styles (pp. 185-228). New York: Plenum.

Biggs, John y Collis, Kevin. (1982). Evaluating the Quality of Learning: The SOLO Taxonomy. New York: Plenum Press. 
Biggs, John, Lai, Patrick, Tang, Catherine y Lavelle, Ellen. (1999). The effect of a graduate workshop on graduate students writing in English as a second language. British Journal of Educational Psychology, 69, 293-306.

Boscolo, Pietro, Arfé, Barbara y Quarisa, Mara. (2007). Improving the quality of students' academic writing: an intervention study. Studies in Higher Education, 32(4), 419-438.

Campbell, Jennifer, Smith, David y Brooker, Ross. (1998). From conception to performance: How undergraduate students conceptualize and construct essays. Higher Education, 36, 449-469.

Carlino, Paula. (2004). El proceso de escritura académica: cuatro dificultades de la enseñanza universitaria. Educere, 8(26), 321-327.

Carlino, Paula. (2005). La experiencia de escribir una tesis: contextos que la vuelven más difícil. Anales del Instituto de Lingüística, XXVI, 41-62.

Cassany, Daniel y Morales, Oscar. (2009). Leer y escribir en la universidad: los géneros científicos. En Daniel Cassany (Comp.), Para ser letrados (pp. 109-128). Barcelona: Paidós.

Difabio de Anglat, Hilda. (2012). Hacia un inventario de escritura académica en el posgrado. Revista de Orientación Educacional, 26(49), 37-53.

Ellis, Robert, Taylor, Charlotte y Drury, Helen. (2007). Learning science through writing: associations with prior conceptions of writing and perceptions of a writing program. Higher Education Research \& Development, 26(3), 297-311.

Fernández Nistal, M. Teresa, Pérez Ibarra, Ricardo, Peña Boone, Sergio y Mercado Ibarra, S. Magdalena. (2011). Concepciones sobre la enseñanza del profesorado y sus actuaciones en clases de ciencias naturales de educación secundaria. Revista Mexicana de Investigación Educativa, 16(49), 571-596.

Flower, Linda y Hayes, John. (1981). A cognitive process theory of writing. College Composition and Communication, 32(4), 365-387.

Grant, Janet. (2002). Undertaking research in medical education. Open University: Danish Association of Medical Education.

Hernández Rojas, Gerardo. (2012). Teorías implícitas de escritura en estudiantes pertenecientes a dos comunidades académicas distintas. Perfiles Educativos, $X X X I V(136), 42-62$.

Hounsell, Dai. (1984). Learning and essay writing. En Ference Marton, Dai Hounsell y Noel Entwistle (Eds.), The experience of learning (pp.103-123). Edinburgh: Scottish Academic Press.

Lavelle, Ellen. (1993). Development and validation of an inventory to assess processes in college composition. British Journal of Educational Psychology, 63(3), 489-499. 
Lavelle, Ellen y Bushrow, Kathy. (2007). Writing approaches of graduate students. Educational Psychology, 27(6), 807-822.

Lavelle, Ellen y Guarino, Anthony. (2003). A Multidimensional Approach to Understanding College Writing Processes. Educational Psychology, 23(3), 295-305.

Lavelle, Ellen y Zuercher, Nancy. (2001). The writing approaches of university students. Higher Education, 42, 373-391.

Levin, Tamar y Wagner, Tili. (2006). In their own words: Understanding student conceptions of writing through their spontaneous metaphors in the science classroom. Instructional Science, 34(3), 227-278.

Lonka, Kirsti. (1996). The writing process questionnaire. Helsinki: Department of Psychology, University of Helsinki.

Lovitts, Barbara. (2005). Being a good course-taker is not enough: a theoretical perspective on the transition to independent research. Studies in Higher Education, 30(2), 137-154.

Marton, Ference y Säljö, Roger. (1976). On qualitative differences in learning: I-Outcome and Process. British Journal of Educational Psychology, 46, 4-11.

Mateos, Mar, Cuevas, Isabel, Martín, Elena, Martín, Ana, Echeita, Gerardo y Luna, María. (2011). Reading to write an argumentation: the role of epistemological, reading and writing beliefs. Journal of Research in Reading, 34(3), 281-297.

Narvaja de Arnoux, Elvira, Borsinger, Ann, Carlino, Paula, Di Stefano, Mariana, Pereira, Cecilia y Silvestre, Adriana. (2005). La intervención pedagógica en el proceso de escritura de Tesis de Posgrado. Revista de la Maestría en Salud Pública, 3(6), 2-18.

Scardamalia, Marlene y Bereiter, Carl. (1992). Dos modelos explicativos de los procesos de producción escrita. Infancia y aprendizaje, 58, 43-64.

Serrano de Moreno, Stella. (2001). La argumentación como problema en la composición escrita de estudiantes de formación docente. Lectura y Vida, 22(4), 26-37.

Silva, Tony y Nicholls, Jason. (1993). College students as writing theorists: Goals and beliefs about the causes of success. Contemporary Educational Psychology, 18, 281-293.

van Dijk, Teun y Kintsch, Walter. (1983). Strategies of Discourse Comprehension. Orlando: Academic Press.

Vázquez, Alicia y Jacob, Ivone. (2006). Escribir textos académicos en la Universidad: intervención didáctica y enfoques de los estudiantes. En María Zulma Lanz (Comp.), El aprendizaje autorregulado. Enseñar a aprender en diferentes entornos educativos (pp.53-73). Buenos Aires: Noveduc. 
Villalón Molina, Ruth. (2010). Las concepciones de los estudiantes sobre la escritura académica (Tesis para optar por el grado de Doctor en Psicología, Universidad Autónoma de Madrid). Recuperada de http://www.tdx.cat/handle/10803/50700

Villalón Molina, Ruth y Mateos, Mar. (2009). Concepciones del alumnado de secundaria y universidad sobre la escritura académica. Infancia y Aprendizaje, 32(2), 219-232.

Wells, Gordon. (1990). Talk about Text: Where Literacy is Learned and Taught. Curriculum Inquiry, 20(4), 369-405.

White, Mary Jane y Bruning, Roger. (2005). Implicit writing beliefs and their relation to writing quality. Contemporary Educational Psychology, 30(2), 166-189.

Zimmerman, Barry y Bandura, Albert. (1994). Impact of self-regulatory influences on writing course attainment. American Educational Research Journal, 31(4), 845-862. 